\title{
Fundamental solution matrix and Cauchy properties of quaternion combined impulsive matrix dynamic equation on time scales*
}

\author{
Chao Wang, Zhien Li and Ravi P. Agarwal
}

\begin{abstract}
In this paper, we establish some basic results for quaternion combined impulsive matrix dynamic equation on time scales for the first time. Quaternion matrix combined-exponential function is introduced and some basic properties are obtained. Based on this, the fundamental solution matrix and corresponding Cauchy matrix for a class of quaternion matrix dynamic equation with combined derivatives and bi-directional impulses are derived.
\end{abstract}

\section{Introduction}

In 1843, Hamilton initiated the notion of quaternions which extends the complex numbers to the four-dimensional space (see [8]). The multiplication of quaternions are determined by a noncommutative division algebra. Let $q=$ $q_{0}+q_{1} i+q_{2} j+q_{3} k$, be a quaternion, where $q_{0}, q_{1}, q_{2}, q_{3} \in \mathbb{R}$, and $i, j, k$ satisfy the multiplication: $i^{2}=j^{2}=k^{2}=i j k=-1, j k=-k j=i, k i=-i k=j, i j=$ $-j i=k$. In the real world, there exists the quaternionic differential equation structure in many research fields such as differential geometry, fluid mechanics, attitude dynamics, quantum mechanics (see [1]), etc., and many researchers focus on the subject with quaternionic background (see [5-7,10,51-54]).

Key Words: Quaternion; Cauchy matrix; Combined matrix dynamic equation; Bidirectional impulses; Time scales.

2010 Mathematics Subject Classification: 34A37, 34N05, 11R52.

Received: 11.01.2021

Accepted: 19.01.2021

*This work is supported by NSFC (No. 11961077). 
On the other hand, in 1988, Stefan Hilger introduced the theory of time scales, which is a powerful tool to study dynamic equations on hybrid domains (see [9]), by choosing the time scale to be the set of real numbers, the general results yield the results concerning different types of dynamic equations (see $[20,23,27])$. In 2016, some equivalent concepts of periodic time scales were addressed by Wang and Agarwal et al.(see [2,21,22]). In [24,26,28], the concept of piecewise almost periodic and almost automorphic functions on time scales with periodicity was first introduced and applied to analyze the almost periodic solutions to neural networks and biological dynamic models. In [29,31], the authors proposed the $\Pi$-semigroup and the semigroups induced by completeclosed time scales to study the almost periodic mild solutions to evolution equations. Due to the irregularity of time scales, the delay classification was addressed to solve the delay dynamic equations on hybrid time scales (see [30]). The notion of changing-periodic time scales is a recent new concept which can deal with the translation solution problems of the dynamic equations on time scales with the bounded graininess function $\mu$ (see [32]). By using the idea of decomposition of time scales, the existence and stability of local solutions of dynamic equations on piecewise periodic time scales were considered (see $[33,34])$. Moreover, the concept of matched spaces of time scales was put forward to assist in solving the problems on non-translational shift time scales (see [25, 35-37]). By choosing $\mathbb{T}=h \mathbb{Z}$, the same result yields a result for difference equations with $h$-step; let the quantum time scale $\mathbb{T}=q^{\mathbb{Z}}$, the hybrid domains $\mathbb{T}=\{h \mathbb{Z}\} \cup\left\{\overline{q^{\mathbb{Z}}}\right\}$, etc., one can obtain a much more general result by using the theory of time scales (see [3,4,38-41]). In 2020, Wang, Li, Agarwal and O'Regan investigated the commutativity of quaternion-matrixvalued functions and quaternion matrix dynamic equations on time scales and nine interesting problems were proposed and solved (see [13-15]) in which several real applications were demonstrated in applied dynamic equations. In 2020, the coupled-jumping timescale theory and applications were proposed (see $[50])$.

In 2006, Sheng, Fadag, et al. introduced a combined derivative on time scales called diamond derivatives which is defined as a linear combination of Delta and Nabla dynamic derivatives and is a more accurate approximation to the conventional derivative. The calculation $\diamond_{\alpha}$ diamond derivatives of a function needs the function is Delta and Nabla derivable both (see [17, 18]). In particular, for $\alpha=1$ the $\nabla_{\alpha}$ diamond derivatives is $\Delta$-derivatives, and for $\alpha=0$ the $\nabla_{\alpha}$ diamond derivatives is $\nabla$-derivatives. In 2020, Wang and Agarwal et.al established the combined measure theory on time scales and it was applied to study Lebesgue-Stieltjes combined $\nabla_{\alpha}$-measure and integral (see $[16,42])$. In [12], the non-eigenvalue form of Liouville's formula and $\alpha$ matrix exponential solutions for combined matrix dynamic equations on time 
scales were obtained.

However, there is no work on the combined matrix dynamic equations on time scales under quaternionic background. Moreover, the dynamic equations with impulses demonstrate their advantages in describing the dynamical behavior with a sudden change or an impact, it is significant to investigate the impulsive dynamic equations on hybrid domains (see [43-49]). Motivated by the above, since impulsive dynamic equations play a vital role in depicting the natural phenomena with sudden changes in the practical applications (see $[11,19])$, we will introduce a quaternion matrix combined-exponential function and study its properties. Based on it, a class of quaternion matrix dynamic equation with combined derivatives and bi-directional impulses is introduced and investigated, some basic results including the fundamental solution matrix and corresponding Cauchy matrix are obtained for the first time.

\section{Preliminaries}

In what follows, we will present some fundamental knowledge of combined calculus on time scales and generalize these results under the quaternion background. For convenience, we denote quaternion space by $\mathbb{Q}$ throughout the paper.

A time scale $\mathbb{T}$ is a closed subset of $\mathbb{R}$. It follows that the jump operators $\sigma, \rho: \mathbb{T} \rightarrow \mathbb{T}$ defined by $\sigma(t)=\inf \{s \in \mathbb{T}: s>t\}$ and $\rho(t)=\sup \{s \in \mathbb{T}: s<t\}$ (supplemented by $\inf \phi:=\sup \mathbb{T}$ and $\sup \phi:=\inf \mathbb{T}$ ) are well defined. The point $t \in \mathbb{T}$ is left-dense, left-scattered, right-dense, right-scattered if $\rho(t)=$ $t, \rho(t)<t, \sigma(t)=t, \sigma(t)>t$, respectively. If $\mathbb{T}$ has a right scattered minimum $m$, define $\mathbb{T}_{\kappa}:=\mathbb{T} \backslash m$; otherwise, set $\mathbb{T}^{\kappa}=\mathbb{T}$. The notations $[a, b]_{\mathbb{T}},[a, b)_{\mathbb{T}}$ and so on, we will denote time scale intervals $[a, b]_{\mathbb{T}}=\{t \in \mathbb{T}: a \leq t \leq b\}$, where $a, b \in \mathbb{T}$ with $a<\rho(b)$.

Definition 2.1 ( [4]). For $y: \mathbb{T} \rightarrow \mathbb{R}$ and $t \in \mathbb{T}^{\kappa}$, we define the $\Delta$-derivative of $y(t), y^{\Delta}(t)$, to be the number (if it exists) with the property that for a given $\varepsilon>0$, there exists a neighborhood $U$ of $t$ (i.e., $U=(t-\delta, t+\delta)_{\mathbb{T}}$ for some $\delta>0)$ such that

$$
\left|[y(\sigma(t))-y(s)]-y^{\Delta}(t)[\sigma(t)-s]\right|<\varepsilon|\sigma(t)-s|
$$

for all $s \in U$. That is, the limit

$$
y^{\Delta}(t)=\lim _{s \rightarrow t} \frac{f(\sigma(t))-f(s)}{\sigma(t)-s}
$$

exists. 
Theorem 2.1 ( [4]). If $a, b, c \in \mathbb{T}, \alpha, \beta \in \mathbb{R}$, and $f, g \in C_{r d}$, then

(i) $\int_{a}^{b}[\alpha f(t)+\beta g(t)] \Delta t=\alpha \int_{a}^{b} f(t) \Delta t+\beta \int_{a}^{b} g(t) \Delta t$;

(ii) $\int_{a}^{b} f(t) \Delta t=-\int_{b}^{a} f(t) \Delta t$;

(iii) $\int_{a}^{c} f(t) \Delta t=\int_{a}^{b} f(t) \Delta t+\int_{b}^{c} f(t) \Delta t ;$

(iv) $\left|\int_{a}^{b} f(t) \Delta t\right| \leq \int_{a}^{b}|f(t)| \Delta t$.

Definition 2.2 ( [4]). If $r$ is a $\mu$-regressive function, then the generalized exponential function $e_{r}$ is defined by

$$
e_{r}(t, s)=\exp \left\{\int_{s}^{t} \xi_{\mu(\tau)}(r(\tau)) \Delta \tau\right\}
$$

for all $s, t \in \mathbb{T}$, where the $\mu$-cylinder transformation is as in

$$
\xi_{h}(z):=\frac{1}{h} \log (1+z h) .
$$

Theorem 2.2 ( [4]). Assume that $p, q: \mathbb{T} \rightarrow \mathbb{R}$ are two $\mu$-regressive functions.

Then

(i) $e_{0}(t, s) \equiv 1$ and $e_{p}(t, t) \equiv 1$;

(ii) $e_{p}(\sigma(t), s)=(1+\mu(t) p(t)) e_{p}(t, s)$;

(iii) $e_{p}(t, s)=\frac{1}{e_{p}(s, t)}=e_{\ominus p}(s, t)$;

(iv) $e_{p}(t, s) e_{p}(s, r)=e_{p}(t, r)$;

(v) $\left(e_{\ominus p}(t, s)\right)^{\Delta}=(\ominus p)(t) e_{\ominus p}(t, s)$;

Definition $2.3([1])$. Let $A: \mathbb{T} \rightarrow \mathbb{Q}^{n \times n}$ and $A(t)=\left[a_{r h 0}(t)+i a_{r h 1}(t)+\right.$ $\left.j a_{r h 2}(t)+k a_{r h 3}(t)\right]_{n \times n}$, where $1 \leq r, h \leq n, n \in \mathbb{N}^{+}$, the integral of the matrix function $A(t)$ is defined by

$$
\begin{aligned}
& \int_{t_{0}}^{t} A(\tau) \Delta \tau= \\
& {\left[\int_{t_{0}}^{t} a_{r h 0}(\tau) \Delta \tau+i \int_{t_{0}}^{t} a_{r h 1}(\tau) \Delta \tau+j \int_{t_{0}}^{t} a_{r h 2}(\tau) \Delta \tau+k \int_{t_{0}}^{t} a_{r h 3}(\tau) \Delta \tau\right]_{n \times n} .}
\end{aligned}
$$

Definition 2.4 ( $[18])$. Let $A: \mathbb{T} \rightarrow \mathbb{Q}^{n \times n}$ be differentiable on $\mathbb{T}$ in $\Delta$ and $\nabla$ sense. For $t \in \mathbb{T}$, define a diamond- $\alpha$ dynamic derivative $A^{\diamond \alpha}(t)$ by

$$
A^{\diamond_{\alpha}}(t)=\alpha A^{\Delta}(t)+(1-\alpha) A^{\nabla}(t), \quad 0 \leq \alpha \leq 1 .
$$

Thus $A(t)$ is diamond- $\alpha$ differentiable if and only if $A(t)$ is $\Delta$ and $\nabla$ differentiable. 
Consider the following two initial value problems of homogeneous quaternion matrix dynamic equations on time scales.

$$
\begin{gathered}
X^{\nabla}(t)=A(t) X(t), X\left(t_{0}\right)=I, \\
X^{\Delta}(t)=A(t) X(t), \quad X\left(t_{0}\right)=I,
\end{gathered}
$$

where $I$ is an identity matrix.

Now, we will derive a quaternion matrix $\nabla$-exponential function $\hat{e}_{A}\left(t, t_{0}\right)$ by calculating the solutions of the initial value problems of the quaternion matrix $\nabla$-dynamic equation (1). Similarly, a quaternion matrix $\Delta$-exponential function $e_{A}\left(t, t_{0}\right)$ can also be obtained.

Theorem 2.3. The quaternion matrix exponential function $\hat{e}_{A}\left(t, t_{0}\right)$ is given by:

$\hat{e}_{A}\left(t, t_{0}\right)=I+\sum_{n=1}^{\infty} \int_{t_{0}}^{t} A^{\sigma}\left(t_{n}\right) \int_{t_{0}}^{\sigma\left(t_{n}\right)} A^{\sigma}\left(t_{n-1}\right) \ldots \int_{t_{0}}^{\sigma\left(t_{2}\right)} A^{\sigma}\left(t_{1}\right) \Delta t_{1} \ldots \Delta t_{n-1} \Delta t_{n}$ and the quaternion matrix $\Delta$-exponential function $e_{A}\left(t, t_{0}\right)$ is given by:

$$
e_{A}\left(t, t_{0}\right)=I+\sum_{n=1}^{\infty} \int_{t_{0}}^{t} A\left(t_{n}\right) \int_{t_{0}}^{t_{n}} A\left(t_{n-1}\right) \ldots \int_{t_{0}}^{t_{2}} A\left(t_{1}\right) \Delta t_{1} \ldots \Delta t_{n-1} \Delta t_{n}
$$

where $I$ is $n \times n$-identity matrix.

Proof. For $\hat{e}_{A}\left(t, t_{0}\right)$, by calculating the solution of the initial value problem of (1), we can obtain

$$
\begin{aligned}
& \nu(t) \hat{e}_{A}^{\nabla}\left(t, t_{0}\right)=\hat{e}_{A}\left(t, t_{0}\right)-\hat{e}_{A}\left(\rho(t), t_{0}\right) \\
= & I+\sum_{n=1}^{\infty} \int_{t_{0}}^{t} A^{\sigma}\left(t_{n}\right) \int_{t_{0}}^{\sigma\left(t_{n}\right)} A^{\sigma}\left(t_{n-1}\right) \ldots \int_{t_{0}}^{\sigma\left(t_{2}\right)} A^{\sigma}\left(t_{1}\right) \Delta t_{1} \ldots \Delta t_{n-1} \Delta t_{n} \\
& -\left(I+\sum_{n=1}^{\infty} \int_{t_{0}}^{\rho(t)} A^{\sigma}\left(t_{n}\right) \ldots \int_{t_{0}}^{\sigma\left(t_{2}\right)} A^{\sigma}\left(t_{1}\right) \Delta t_{1} \ldots \Delta t_{n}\right) \\
= & \sum_{n=1}^{\infty} \int_{\rho(t)}^{t} A^{\sigma}\left(t_{n}\right) \int_{t_{0}}^{\sigma\left(t_{n}\right)} A^{\sigma}\left(t_{n-1}\right) \ldots \int_{t_{0}}^{\sigma\left(t_{2}\right)} A^{\sigma}\left(t_{1}\right) \Delta t_{1} \ldots \Delta t_{n-1} \Delta t_{n} \\
= & \nu(t) A^{\sigma}(\rho(t))\left(I+\sum_{n=1}^{\infty} \int_{t_{0}}^{\sigma(\rho(t))} A^{\sigma}\left(t_{n}\right) \int_{t_{0}}^{\sigma\left(t_{n}\right)} A^{\sigma}\left(t_{n-1}\right) \ldots \Delta t_{n}\right) \\
= & \nu(t) A(t) \hat{e}_{A}\left(t, t_{0}\right) .
\end{aligned}
$$

Hence $\hat{e}_{A}\left(t, t_{0}\right)$ is the solution of initial value problem of (1). Similarly, through the same proof process, we can obtain (3). The proof is completed. 


\section{Quaternion matrix diamond-exponential function and quaternion combined matrix dynamic equation}

In the sequel, we will introduce a definition of quaternion matrix diamondexponential function through matrix exponential functions introduced in Theorem 2.3. For convenience, we introduce some notations, $e_{A}\left(\rho(t), t_{0}\right):=$ $e_{A}^{\rho}\left(t, t_{0}\right), A(\rho(t))=A^{\rho}(t), e_{A}\left(\sigma(t), t_{0}\right):=e_{A}^{\sigma}\left(t, t_{0}\right), A(\sigma(t))=A^{\sigma}(t)$, so does $\hat{e}$.

Definition 3.1. Let $A: \mathbb{T} \rightarrow \mathbb{Q}^{n \times n}$, where $n \in \mathbb{N}^{+}$, we define a quaternion matrix diamond-exponential function by $\dot{e}_{A}=\alpha e_{A}\left(t, t_{0}\right)+(1-\alpha) \hat{e}_{A}\left(t, t_{0}\right)$.

Lemma 3.1. $e_{A}^{\nabla}\left(t, t_{0}\right)=A^{\rho}(t)\left(I+\nu(t) A^{\rho}(t)\right)^{-1} e_{A}\left(t, t_{0}\right)$ if $I+\nu(t) A^{\rho}(t)$ is invertible.

Proof. By Theorem 2.3, we have

$$
\begin{aligned}
e_{A}^{\nabla}\left(t, t_{0}\right) & =\frac{e_{A}\left(t, t_{0}\right)-e_{A}^{\rho}\left(t, t_{0}\right)}{\nu(t)} \\
& =\frac{\sum_{n=1}^{\infty} \int_{\rho(t)}^{t} A\left(t_{n}\right) \int_{t_{0}}^{t_{n}} A\left(t_{n-1}\right) \ldots \int_{t_{0}}^{t_{2}} A\left(t_{1}\right) \Delta t_{1} \ldots \Delta t_{n-1} \Delta t_{n}}{\nu(t)} \\
& =A(\rho(t))\left(I+\sum_{n=1}^{\infty} \int_{t_{0}}^{\rho(t)} A\left(t_{n}\right) \ldots \int_{t_{0}}^{t_{2}} A\left(t_{1}\right) \Delta t_{1} \ldots \Delta t_{n}\right) \\
& =A(\rho(t)) e_{A}\left(\rho(t), t_{0}\right) .
\end{aligned}
$$

On the other hand, since

$$
e_{A}\left(t, t_{0}\right)=e_{A}^{\rho}\left(t, t_{0}\right)+\nu(t) e_{A}^{\nabla}\left(t, t_{0}\right)=(I+\nu(t) A(\rho(t))) e_{A}\left(\rho(t), t_{0}\right),
$$

then $e_{A}\left(\rho(t), t_{0}\right)=(I+\nu(t) A(\rho(t)))^{-1} e_{A}\left(t, t_{0}\right)$. Therefore $e_{A}^{\nabla}\left(t, t_{0}\right)=A^{\rho}(t)(I+$ $\left.\nu(t) A^{\rho}(t)\right)^{-1} e_{A}\left(t, t_{0}\right)$. The proof is completed.

Lemma 3.2. $\hat{e}_{A}^{\Delta}\left(t, t_{0}\right)=A^{\sigma}(t)\left(I-\mu(t) A^{\sigma}(t)\right)^{-1} \hat{e}_{A}\left(t, t_{0}\right)$ if $I-\mu(t) A^{\sigma}(t)$ is invertible.

Proof. By Theorem 2.3, we have

$$
\begin{aligned}
& \hat{e}_{A}^{\Delta}\left(t, t_{0}\right)=\frac{\hat{e}_{A}\left(\sigma(t), t_{0}\right)-\hat{e}_{A}\left(t, t_{0}\right)}{\mu(t)} \\
= & \frac{\sum_{n=1}^{\infty} \int_{t}^{\sigma(t)} A\left(\sigma\left(t_{n}\right)\right) \int_{t_{0}}^{\sigma\left(t_{n}\right)} A\left(\sigma\left(t_{n-1}\right)\right) \ldots \int_{t_{0}}^{\sigma\left(t_{2}\right)} A\left(\sigma\left(t_{1}\right)\right) \Delta t_{1} \ldots \Delta t_{n-1} \Delta t_{n}}{\mu(t)}
\end{aligned}
$$




$$
\begin{aligned}
& =A(\sigma(t))\left(I+\sum_{n=1}^{\infty} \int_{t_{0}}^{\sigma(t)} A\left(\sigma\left(t_{n}\right)\right) \ldots \int_{t_{0}}^{\sigma\left(t_{2}\right)} A\left(\sigma\left(t_{1}\right)\right) \Delta t_{1} \ldots \Delta t_{n}\right) \\
& =A(\sigma(t)) \hat{e}_{A}^{\sigma}\left(t, t_{0}\right) .
\end{aligned}
$$

On the other hand, since

$$
\hat{e}_{A}^{\sigma}\left(t, t_{0}\right)=\hat{e}_{A}\left(t, t_{0}\right)+\mu(t) \hat{e}_{A}^{\Delta}\left(t, t_{0}\right),
$$

then $\hat{e}_{A}^{\sigma}\left(t, t_{0}\right)=\left(I-\mu(t) A^{\sigma}(t)\right)^{-1} \hat{e}_{A}\left(t, t_{0}\right)$. Therefore $\hat{e}_{A}^{\Delta}\left(t, t_{0}\right)=A^{\sigma}(t)(I-$ $\left.\mu(t) A^{\sigma}(t)\right)^{-1} \hat{e}_{A}\left(t, t_{0}\right)$. The proof is completed.

Now, consider the following quaternion combined matrix dynamic equation:

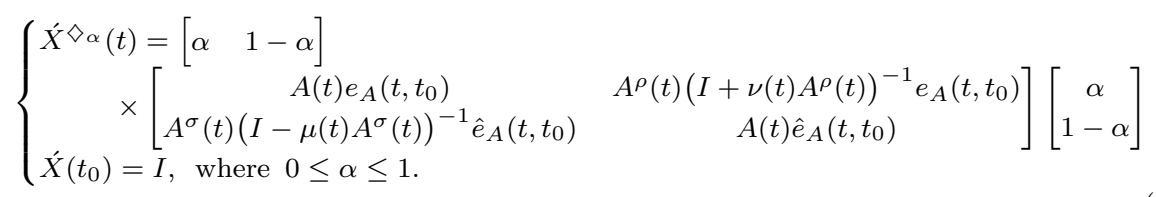

Remark 3.1. Note that the quaternion combined matrix dynamic equation (1) will turn into (1) for $\alpha=0$; moreover, it will turn into (2) for $\alpha=1$.

Theorem 3.1. $e_{A}\left(t, t_{0}\right)$ is the matrix exponential solution of the initial value problem of (1).

Proof. By Theorem 2.3, Lemma 3.1 and Definition 2.4, we obtain

$$
e_{A}^{\diamond_{\alpha}^{\alpha}}\left(t, t_{0}\right)=\left(\alpha A(t)+(1-\alpha) A^{\rho}(t)\left(I+\nu(t) A^{\rho}(t)\right)^{-1}\right) e_{A}\left(t, t_{0}\right) .
$$

Similarly, by Theorem 2.3, Lemma 3.2 and Definition 2.4, we have

$$
\hat{e}_{A}^{\diamond_{\alpha}^{\alpha}}\left(t, t_{0}\right)=\left(\alpha A^{\sigma}(t)\left(I-\mu(t) A^{\sigma}(t)\right)^{-1}+(1-\alpha) A(t)\right) \hat{e}_{A}\left(t, t_{0}\right) .
$$

By Definitions 2.4 and 3.1, we have

$$
\begin{aligned}
\dot{e}_{A}^{\diamond_{\alpha}}\left(t, t_{0}\right)= & \left(\alpha A(t)+(1-\alpha) A^{\rho}(t)\left(I+\nu(t) A^{\rho}(t)\right)^{-1}\right) \alpha e_{A}\left(t, t_{0}\right) \\
& +\left(\alpha A^{\sigma}(t)\left(I-\mu(t) A^{\sigma}(t)\right)^{-1}+(1-\alpha) A(t)\right)(1-\alpha) \hat{e}_{A}\left(t, t_{0}\right) .
\end{aligned}
$$

Therefore, we can obtain the desired result. 
Now, we consider the following non-homogeneous quaternion matrix $\Delta$ dynamic equation:

$$
\left\{\begin{array}{l}
X^{\Delta}(t)=A(t) X(t)+H(t) \\
X\left(t_{0}\right)=X_{0},
\end{array}\right.
$$

where $A, H: \mathbb{T} \rightarrow \mathbb{Q}^{n \times n}, X_{0} \in \mathbb{Q}^{n \times n}, n \in \mathbb{N}^{+}$.

Theorem 3.2. The fundamental solution matrix of (2) is given by

$$
\begin{gathered}
X(t)= \\
\left(I+\sum_{n=1}^{\infty} \int_{t_{0}}^{t} A\left(\tau_{n}\right) \int_{t_{0}}^{\tau_{n}} A\left(\tau_{n-1}\right) \ldots \int_{t_{0}}^{\tau_{2}} A\left(\tau_{1}\right) \Delta \tau_{1} \ldots \Delta \tau_{n-1} \Delta \tau_{n}\right) X_{0} \\
+\int_{t_{0}}^{t}\left(I+\sum_{n=1}^{\infty} \int_{\sigma(\tau)}^{t} A\left(\tau_{n}\right) \ldots \int_{\sigma(\tau)}^{\tau_{2}} A\left(\tau_{1}\right) \Delta \tau_{1} \ldots \Delta \tau_{n}\right) H(\tau) \Delta \tau, \\
\text { i.e., } X(t)=e_{A}\left(t, t_{0}\right) X_{0}+\int_{t_{0}}^{t} e_{A}(t, \sigma(\tau)) H(\tau) \Delta \tau .
\end{gathered}
$$

Proof. For (3), we have

$$
\begin{aligned}
& \mu(t) X^{\Delta}(t) \\
&=\left(I+\sum_{n=1}^{\infty} \int_{t_{0}}^{\sigma(t)} A\left(\tau_{n}\right) \int_{t_{0}}^{\tau_{n}} A\left(\tau_{n-1}\right) \ldots \int_{t_{0}}^{\tau_{2}} A\left(\tau_{1}\right) \Delta \tau_{1} \ldots \Delta \tau_{n-1} \Delta \tau_{n}\right) X_{0} \\
&+\int_{t_{0}}^{\sigma(t)}\left(I+\sum_{n=1}^{\infty} \int_{\sigma(\tau)}^{\sigma(t)} A\left(\tau_{n}\right) \ldots \int_{\sigma(\tau)}^{\tau_{2}} A\left(\tau_{1}\right) \Delta \tau_{1} \ldots \Delta \tau_{n}\right) H(\tau) \Delta \tau \\
&-\left(I+\sum_{n=1}^{\infty} \int_{t_{0}}^{t} A\left(\tau_{n}\right) \int_{t_{0}}^{\tau_{n}} A\left(\tau_{n-1}\right) \ldots \int_{t_{0}}^{\tau_{2}} A\left(\tau_{1}\right) \Delta \tau_{1} \ldots \Delta \tau_{n-1} \Delta \tau_{n}\right) X_{0} \\
&-\int_{t_{0}}^{t}\left(I+\sum_{n=1}^{\infty} \int_{\sigma(\tau)}^{t} A\left(\tau_{n}\right) \ldots \int_{\sigma(\tau)}^{\tau_{2}} A\left(\tau_{1}\right) \Delta \tau_{1} \ldots \Delta \tau_{n}\right) H(\tau) \Delta \tau \\
&= \mu(t) A(t)\left(I+\sum_{n=1}^{\infty} \int_{t_{0}}^{t} A\left(\tau_{n}\right) \int_{t_{0}}^{\tau_{n}} A\left(\tau_{n-1}\right) \ldots \int_{t_{0}}^{\tau_{2}} A\left(\tau_{1}\right) \Delta \tau_{1} \ldots \Delta \tau_{n-1} \Delta \tau_{n}\right) \\
& \times X_{0}+\int_{t_{0}}^{t}\left(\sum_{n=1}^{\infty} \int_{t}^{\sigma(t)} A\left(\tau_{n}\right) \ldots \int_{\sigma(\tau)}^{\tau_{2}} A\left(\tau_{1}\right) \Delta \tau_{1} \ldots \Delta \tau_{n}\right) H(\tau) \Delta \tau \\
&+\int_{t}^{\sigma(t)}\left(I+\sum_{n=1}^{\infty} \int_{\sigma(\tau)}^{\sigma(t)} A\left(\tau_{n}\right) \ldots \int_{\sigma(\tau)}^{\tau_{2}} A\left(\tau_{1}\right) \Delta \tau_{1} \ldots \Delta \tau_{n}\right) H(\tau) \Delta \tau \\
& \mu(t) A(t)\left(I+\sum_{n=1}^{\infty} \int_{t_{0}}^{t} A\left(\tau_{n}\right) \int_{t_{0}}^{\tau_{n}} A\left(\tau_{n-1}\right) \ldots \int_{t_{0}}^{\tau_{2}} A\left(\tau_{1}\right) \Delta \tau_{1} \ldots \Delta \tau_{n-1} \Delta \tau_{n}\right) \\
&=
\end{aligned}
$$




$$
\begin{aligned}
& \times X_{0}+\mu(t) A(t) \int_{t_{0}}^{t}\left(I+\sum_{n=1}^{\infty} \int_{\sigma(\tau)}^{t} A\left(\tau_{n}\right) \ldots \int_{\sigma(\tau)}^{\tau_{2}} A\left(\tau_{1}\right) \Delta \tau_{1} \ldots \Delta \tau_{n}\right) \\
& \times H(\tau) \Delta \tau+\mu(t) H(t)=\mu(t) A(t) X(t)+\mu(t) H(t) .
\end{aligned}
$$

Thus we can obtain the desired result.

Consider the following non-homogeneous quaternion matrix $\nabla$-dynamic equation:

$$
\left\{\begin{array}{l}
\tilde{X}^{\nabla}(t)=A(t) \tilde{X}(t)+H(t) \\
\tilde{X}\left(t_{0}\right)=X_{0}
\end{array}\right.
$$

where $A, H: \mathbb{T} \rightarrow \mathbb{Q}^{n \times n}, X_{0} \in \mathbb{Q}^{n \times n}, n \in \mathbb{N}^{+}$.

Theorem 3.3. The fundamental solution matrix of (4) is given as

$$
\begin{aligned}
& \tilde{X}(t)= \\
& \left(I+\sum_{n=1}^{\infty} \int_{t_{0}}^{t} A^{\sigma}\left(\tau_{n}\right) \int_{t_{0}}^{\sigma\left(\tau_{n}\right)} A^{\sigma}\left(\tau_{n-1}\right) \ldots \int_{t_{0}}^{\sigma\left(\tau_{2}\right)} A^{\sigma}\left(\tau_{1}\right) \Delta \tau_{1} \ldots \Delta \tau_{n-1} \Delta \tau_{n}\right) \\
& \times X_{0}+\int_{t_{0}}^{t}\left(I+\sum_{n=1}^{\infty} \int_{\tau}^{t} A^{\sigma}\left(\tau_{n}\right) \int_{\tau}^{\sigma\left(\tau_{2}\right)} A^{\sigma}\left(\tau_{1}\right) \Delta \tau_{1} \ldots \Delta \tau_{n}\right) H^{\sigma}(\tau) \Delta \tau, \quad \text { (5) } \\
& \text { i.e., } \tilde{X}(t)=\hat{e}_{A}\left(t, t_{0}\right) X_{0}+\int_{t_{0}}^{t} \hat{e}_{A}(t, \tau) H^{\sigma}(\tau) \Delta \tau .
\end{aligned}
$$

Proof. For (5), we have

$$
\begin{aligned}
& \nu(t) \tilde{X}^{\nabla}(t) \\
= & \left(I+\sum_{n=1}^{\infty} \int_{t_{0}}^{t} A^{\sigma}\left(\tau_{n}\right) \int_{t_{0}}^{\sigma\left(\tau_{2}\right)} A^{\sigma}\left(\tau_{1}\right) \Delta \tau_{1} \ldots \Delta \tau_{n}\right) X_{0} \\
& +\int_{t_{0}}^{t}\left(I+\sum_{n=1}^{\infty} \int_{\tau}^{t} A^{\sigma}\left(\tau_{n}\right) \ldots \int_{\tau}^{\sigma\left(\tau_{2}\right)} A^{\sigma}\left(\tau_{1}\right) \Delta \tau_{1} \ldots \Delta \tau_{n}\right) H^{\sigma}(\tau) \Delta \tau \\
& -\left(I+\sum_{n=1}^{\infty} \int_{t_{0}}^{\rho(t)} A^{\sigma}\left(\tau_{n}\right) \int_{t_{0}}^{\sigma\left(\tau_{2}\right)} A^{\sigma}\left(\tau_{1}\right) \Delta \tau_{1} \ldots \Delta \tau_{n}\right) X_{0} \\
& -\int_{t_{0}}^{\rho(t)}\left(I+\sum_{n=1}^{\infty} \int_{\tau}^{\rho(t)} A^{\sigma}\left(\tau_{n}\right) \ldots \int_{\tau}^{\sigma\left(\tau_{2}\right)} A^{\sigma}\left(\tau_{1}\right) \Delta \tau_{1} \ldots \Delta \tau_{n}\right) H^{\sigma}(\tau) \Delta \tau \\
= & \nu(t) A(t)\left(I+\sum_{n=1}^{\infty} \int_{t_{0}}^{t} A^{\sigma}\left(\tau_{n}\right) \ldots \int_{t_{0}}^{\sigma\left(\tau_{2}\right)} A^{\sigma}\left(\tau_{1}\right) \Delta \tau_{1} \ldots \Delta \tau_{n}\right) X_{0} \\
& +\int_{t_{0}}^{t}\left(\sum_{n=1}^{\infty} \int_{\rho(t)}^{t} A^{\sigma}\left(\tau_{n}\right) \ldots \int_{\tau}^{\sigma\left(\tau_{2}\right)} A^{\sigma}\left(\tau_{1}\right) \Delta \tau_{1} \ldots \Delta \tau_{n}\right) H^{\sigma}(\tau) \Delta \tau \\
& +\int_{\rho(t)}^{t}\left(I+\sum_{n=1}^{\infty} \int_{\tau}^{\rho(t)} A^{\sigma}\left(\tau_{n}\right) \ldots \int_{\tau}^{\sigma\left(\tau_{2}\right)} A^{\sigma}\left(\tau_{1}\right) \Delta \tau_{1} \ldots \Delta \tau_{n}\right) H^{\sigma}(\tau) \Delta \tau
\end{aligned}
$$




$$
\begin{aligned}
= & \nu(t) A(t)\left(I+\sum_{n=1}^{\infty} \int_{t_{0}}^{t} A^{\sigma}\left(\tau_{n}\right) \ldots \int_{t_{0}}^{\sigma\left(\tau_{2}\right)} A^{\sigma}\left(\tau_{1}\right) \Delta \tau_{1} \ldots \Delta \tau_{n}\right) X_{0} \\
& +\nu(t) A(t) \int_{t_{0}}^{t}\left(I+\sum_{n=1}^{\infty} \int_{\tau}^{t} A^{\sigma}\left(\tau_{n}\right) \ldots \int_{\tau}^{\sigma\left(\tau_{2}\right)} A^{\sigma}\left(\tau_{1}\right) \Delta \tau_{1} \ldots \Delta \tau_{n}\right) \\
& \times H^{\sigma}(\tau) \Delta \tau+\nu(t) H^{\sigma}(\rho(t))=\nu(t) A(t) \tilde{X}(t)+\nu(t) H(t) .
\end{aligned}
$$

Then we can obtain the desired result. The proof is completed.

Lemma 3.3. For (3), the following equality holds:

$$
\begin{aligned}
& X^{\nabla}(t)= \\
& A^{\rho(t)}\left(I+\sum_{n=1}^{\infty} \int_{t_{0}}^{\rho(t)} A\left(\tau_{n}\right) \int_{t_{0}}^{\tau_{n}} A\left(\tau_{n-1}\right) \ldots \int_{t_{0}}^{\tau_{2}} A\left(\tau_{1}\right) \Delta \tau_{1} \ldots \Delta \tau_{n-1} \Delta \tau_{n}\right) \\
& \times X_{0}+\int_{\rho(t)}^{t}\left(I+\sum_{n=1}^{\infty} \int_{\sigma(\tau)}^{\rho(t)} A\left(\tau_{n}\right) \ldots \int_{\sigma(\tau)}^{\tau_{2}} A\left(\tau_{1}\right) \Delta \tau_{1} \ldots \Delta \tau_{n}\right) H(\tau) \Delta \tau \\
& +\int_{t_{0}}^{\rho(t)} A^{\rho}(t)\left(I+\sum_{n=1}^{\infty} \int_{\sigma(\tau)}^{\rho(t)} A\left(\tau_{n}\right) \ldots \int_{\sigma(\tau)}^{\tau_{2}} A\left(\tau_{1}\right) \Delta \tau_{1} \ldots \Delta \tau_{n}\right) H(\tau) \Delta \tau,
\end{aligned}
$$

i.e., $X^{\nabla}(t)=A^{\rho}(t) X^{\rho}(t)+H^{\rho}(t)$.

Proof. For (3), we have

$$
\begin{aligned}
& \nu(t) X^{\nabla}(t) \\
= & \left(I+\sum_{n=1}^{\infty} \int_{t_{0}}^{t} A\left(\tau_{n}\right) \int_{t_{0}}^{\tau_{n}} A\left(\tau_{n-1}\right) \ldots \int_{t_{0}}^{\tau_{2}} A\left(\tau_{1}\right) \Delta \tau_{1} \ldots \Delta \tau_{n-1} \Delta \tau_{n}\right) X_{0} \\
& +\int_{t_{0}}^{t}\left(I+\sum_{n=1}^{\infty} \int_{\sigma(\tau)}^{t} A\left(\tau_{n}\right) \ldots \int_{\sigma(\tau)}^{\tau_{2}} A\left(\tau_{1}\right) \Delta \tau_{1} \ldots \Delta \tau_{n}\right) H(\tau) \Delta \tau \\
& -\left(I+\sum_{n=1}^{\infty} \int_{t_{0}}^{\rho(t)} A\left(\tau_{n}\right) \int_{t_{0}}^{\tau_{n}} A\left(\tau_{n-1}\right) \ldots \int_{t_{0}}^{\tau_{2}} A\left(\tau_{1}\right) \Delta \tau_{1} \ldots \Delta \tau_{n-1} \Delta \tau_{n}\right) X_{0} \\
& -\int_{t_{0}}^{\rho(t)}\left(I+\sum_{n=1}^{\infty} \int_{\sigma(\tau)}^{\rho(t)} A\left(\tau_{n}\right) \ldots \int_{\sigma(\tau)}^{\tau_{2}} A\left(\tau_{1}\right) \Delta \tau_{1} \ldots \Delta \tau_{n}\right) H(\tau) \Delta \tau \\
= & \left(\sum_{n=1}^{\infty} \int_{\rho(t)}^{t} A\left(\tau_{n}\right) \int_{t_{0}}^{\tau_{n}} A\left(\tau_{n-1}\right) \ldots \int_{t_{0}}^{\tau_{2}} A\left(\tau_{1}\right) \Delta \tau_{1} \ldots \Delta \tau_{n-1} \Delta \tau_{n}\right) X_{0} \\
& +\int_{\rho(t)}^{t}\left(I+\sum_{n=1}^{\infty} \int_{\sigma(\tau)}^{t} A\left(\tau_{n}\right) \ldots \int_{\sigma(\tau)}^{\tau_{2}} A\left(\tau_{1}\right) \Delta \tau_{1} \ldots \Delta \tau_{n}\right) H(\tau) \Delta \tau \\
& +\int_{t_{0}}^{\rho(t)}\left(\sum_{n=1}^{\infty} \int_{\rho(t)}^{t} A\left(\tau_{n}\right) \ldots \int_{\sigma(\tau)}^{\tau_{2}} A\left(\tau_{1}\right) \Delta \tau_{1} \ldots \Delta \tau_{n}\right) H(\tau) \Delta \tau \\
= & \nu(t) A^{\rho}(t)\left(I+\sum_{n=1}^{\infty} \int_{t_{0}}^{\rho(t)} A\left(\tau_{n}\right) \ldots \int_{t_{0}}^{\tau_{2}} A\left(\tau_{1}\right) \Delta \tau_{1} \ldots \Delta \tau_{n}\right) X_{0}
\end{aligned}
$$




$$
\begin{aligned}
& +\nu(t)\left(I+\sum_{n=1}^{\infty} \int_{\sigma(\rho(t))}^{t} A\left(\tau_{n}\right) \ldots \int_{\sigma(\rho(t))}^{\tau_{2}} A\left(\tau_{1}\right) \Delta \tau_{1} \ldots \Delta \tau_{n}\right) H(\rho(t)) \\
& +\int_{t_{0}}^{\rho(t)} A^{\rho}(t) \nu(t)\left(I+\sum_{n=1}^{\infty} \int_{\sigma(t)}^{\rho(t)} A\left(\tau_{n}\right) \ldots \int_{\sigma(\tau)}^{\tau_{2}} A\left(\tau_{1}\right) \Delta \tau_{1} \ldots \Delta \tau_{n}\right) \\
& \times H(\tau) \Delta \tau=\nu(t) A^{\rho}(t) X^{\rho}(t)+\nu(t) H^{\rho}(t) .
\end{aligned}
$$

The proof is completed.

Lemma 3.4. For (5), the following equality holds:

$$
\begin{aligned}
& \tilde{X}^{\Delta}(t)= \\
& A^{\sigma}(t)\left(I+\sum_{n=1}^{\infty} \int_{t_{0}}^{\sigma(t)} A^{\sigma}\left(\tau_{n}\right) \int_{t_{0}}^{\sigma\left(\tau_{n}\right)} A^{\sigma}\left(\tau_{n-1}\right) \ldots \int_{t_{0}}^{\sigma\left(\tau_{2}\right)} A^{\sigma}\left(\tau_{1}\right) \Delta \tau_{1} \ldots \Delta \tau_{n-1} \Delta \tau_{n}\right) \\
& \times X_{0}+\int_{t_{0}}^{\sigma(t)}\left(\sum_{n=1}^{\infty} \int_{t}^{\sigma(t)} A^{\sigma}\left(\tau_{n}\right) \ldots \int_{\tau}^{\sigma\left(\tau_{2}\right)} A^{\sigma}\left(\tau_{1}\right) \Delta \tau_{1} \ldots \Delta \tau_{n}\right) \\
& \times H^{\sigma}(\tau) \Delta \tau+\int_{t}^{\sigma(t)}\left(I+\sum_{n=1}^{\infty} \int_{\tau}^{t} A^{\sigma}\left(\tau_{n}\right) \ldots \int_{\tau}^{\sigma\left(\tau_{2}\right)} A^{\sigma}\left(\tau_{1}\right) \Delta \tau_{1} \ldots \Delta \tau_{n}\right) H^{\sigma}(\tau) \Delta \tau, \\
& \text { i.e., } \tilde{X}^{\Delta}(t)=A^{\sigma}(t) \tilde{X}^{\sigma}(t)+H^{\sigma}(t) .
\end{aligned}
$$

Proof. For (5), we have

$$
\begin{aligned}
& \mu(t) \tilde{X}^{\Delta}(t) \\
= & \left(I+\sum_{n=1}^{\infty} \int_{t_{0}}^{\sigma(t)} A^{\sigma}\left(\tau_{n}\right) \ldots \int_{t_{0}}^{\sigma\left(\tau_{2}\right)} A^{\sigma}\left(\tau_{1}\right) \Delta \tau_{1} \ldots \Delta \tau_{n}\right) X_{0} \\
& +\int_{t_{0}}^{\sigma(t)}\left(I+\sum_{n=1}^{\infty} \int_{\tau}^{\sigma(t)} A^{\sigma}\left(\tau_{n}\right) \ldots \int_{\tau}^{\sigma\left(\tau_{2}\right)} A^{\sigma}\left(\tau_{1}\right) \Delta \tau_{1} \ldots \Delta \tau_{n}\right) H^{\sigma}(\tau) \Delta \tau \\
& -\left(I+\sum_{n=1}^{\infty} \int_{t_{0}}^{t} A^{\sigma}\left(\tau_{n}\right) \ldots \int_{t_{0}}^{\sigma\left(\tau_{2}\right)} A^{\sigma}\left(\tau_{1}\right) \Delta \tau_{1} \ldots \Delta \tau_{n}\right) X_{0} \\
& -\int_{t_{0}}^{t}\left(I+\sum_{n=1}^{\infty} \int_{\tau}^{t} A^{\sigma}\left(\tau_{n}\right) \ldots \int_{\tau}^{\sigma\left(\tau_{2}\right)} A^{\sigma}\left(\tau_{1}\right) \Delta \tau_{1} \ldots \Delta \tau_{n}\right) H^{\sigma}(\tau) \Delta \tau \\
= & \left(\sum_{n=1}^{\infty} \int_{t}^{\sigma(t)} A^{\sigma}\left(\tau_{n}\right) \ldots \int_{t_{0}}^{\sigma\left(\tau_{2}\right)} A^{\sigma}\left(\tau_{1}\right) \Delta \tau_{1} \ldots \Delta \tau_{n}\right) X_{0} \\
& +\int_{t_{0}}^{\sigma(t)}\left(\sum_{n=1}^{\infty} \int_{t}^{\sigma(t)} A^{\sigma}\left(\tau_{n}\right) \ldots \int_{\tau}^{\sigma\left(\tau_{2}\right)} A^{\sigma}\left(\tau_{1}\right) \Delta \tau_{1} \ldots \Delta \tau_{n}\right) H^{\sigma}(\tau) \Delta \tau \\
& +\int_{t}^{\sigma(t)}\left(I+\sum_{n=1}^{\infty} \int_{\tau}^{t} A^{\sigma}\left(\tau_{n}\right) \ldots \int_{\tau}^{\sigma\left(\tau_{2}\right)} A^{\sigma}\left(\tau_{1}\right) \Delta \tau_{1} \ldots \Delta \tau_{n}\right) H^{\sigma}(\tau) \Delta \tau \\
= & \mu(t) A^{\sigma}(t)\left(I+\sum_{n=1}^{\infty} \int_{t_{0}}^{\sigma(t)} A^{\sigma}\left(\tau_{n}\right) \ldots \int_{t_{0}}^{\sigma\left(\tau_{2}\right)} A^{\sigma}\left(\tau_{1}\right) \Delta \tau_{1} \ldots \Delta \tau_{n}\right) X_{0} \\
& +\mu(t) A^{\sigma}(t) \int_{t_{0}}^{\sigma(t)}\left(I+\sum_{n=1}^{\infty} \int_{\tau}^{\sigma(t)} A^{\sigma}\left(\tau_{n}\right) \ldots \int_{\tau}^{\sigma\left(\tau_{2}\right)} A^{\sigma}\left(\tau_{1}\right) \Delta \tau_{1} \ldots \Delta \tau_{n}\right)
\end{aligned}
$$




$$
\begin{aligned}
& \times H^{\sigma}(\tau) \Delta \tau+\mu(t)\left(I+\sum_{n=1}^{\infty} \int_{t}^{t} A^{\sigma}\left(\tau_{n}\right) \ldots \int_{t}^{\sigma\left(\tau_{2}\right)} A^{\sigma}\left(\tau_{1}\right) \Delta \tau_{1} \ldots \Delta \tau_{n}\right) \\
& \times H^{\sigma}(t)=\mu(t) A^{\sigma}(t) \tilde{X}^{\sigma}(t)+\mu(t) H^{\sigma}(t) .
\end{aligned}
$$

The proof is completed.

Based on Theorems 3.2-3.3 and Lemmas 3.3-3.4, we can consider the following non-homogeneous quaternion combined matrix dynamic equation on time scales:

$$
\left\{\begin{array}{l}
\dot{X}^{\diamond_{\alpha}}(t)=\left[\begin{array}{ll}
\alpha & 1-\alpha
\end{array}\right]\left[\begin{array}{cc}
A(t) X(t)+H(t) & A^{\rho}(t) X^{\rho}(t)+H^{\rho}(t) \\
A^{\sigma}(t) \tilde{X}^{\sigma}(t)+H^{\sigma}(t) & A(t) \tilde{X}(t)+H(t)
\end{array}\right]\left[\begin{array}{c}
\alpha \\
1-\alpha
\end{array}\right] \\
\dot{X}\left(t_{0}\right)=X_{0},
\end{array}\right.
$$

where $0 \leq \alpha \leq 1, A, H: \mathbb{T} \rightarrow \mathbb{Q}^{n \times n}, X_{0} \in \mathbb{Q}^{n \times n}, n \in \mathbb{N}^{+}$.

Theorem 3.4. The fundamental solution matrix of (6) is given by

$$
\dot{X}(t)=\alpha X(t)+(1-\alpha) \tilde{X}(t), \quad 0 \leq \alpha \leq 1 .
$$

Proof. From Theorem 3.2 and Lemma 3.3, we have

$$
X^{\diamond_{\alpha}}(t)=\alpha(A(t) X(t)+H(t))+(1-\alpha)\left(A^{\rho}(t) X^{\rho}(t)+H^{\rho}(t)\right) .
$$

According to Theorem 3.3 and Lemma 3.4, we can obtain

$$
\tilde{X}^{\diamond_{\alpha}}(t)=\alpha\left(A^{\sigma}(t) \tilde{X}^{\sigma}(t)+H^{\sigma}(t)\right)+(1-\alpha)(A(t) \tilde{X}(t)+H(t)) .
$$

Hence

$$
\begin{aligned}
& \dot{X}^{\diamond_{\alpha}}(t)=\alpha X^{\diamond_{\alpha}}(t)+(1-\alpha) \tilde{X}^{\diamond_{\alpha}}(t)= \\
& {\left[\begin{array}{ll}
\alpha & 1-\alpha
\end{array}\right]\left[\begin{array}{cc}
A(t) X(t)+H(t) & A^{\rho}(t) X^{\rho}(t)+H^{\rho}(t) \\
A^{\sigma}(t) \tilde{X}^{\sigma}(t)+H^{\sigma}(t) & A(t) \tilde{X}(t)+H(t)
\end{array}\right]\left[\begin{array}{c}
\alpha \\
1-\alpha
\end{array}\right] .}
\end{aligned}
$$

The proof is completed.

Remark 3.2. In Theorem 3.4, X́(t) can also be written as

$$
\dot{X}(t)=\dot{e}_{A}\left(t, t_{0}\right) X_{0}+\alpha \int_{t_{0}}^{t} e_{A}(t, \sigma(\tau)) H(\tau) \Delta \tau+(1-\alpha) \int_{t_{0}}^{t} \hat{e}_{A}(t, \tau) H^{\sigma}(\tau) \Delta \tau .
$$




\section{Quaternion impulsive non-homogeneous matrix com- bined dynamic equation}

In this section, we will derive the fundamental solution matrix and Cauchy matrix of quaternion impulsive non-homogeneous matrix combined dynamic equations.

Consider a quaternion impulsive non-homogeneous matrix combined dynamic equation as follows:

$$
\left\{\begin{aligned}
\dot{X}^{\diamond_{\alpha}}(t)= & {\left[\begin{array}{ll}
\alpha & 1-\alpha
\end{array}\right]\left[\begin{array}{cc}
A(t) X(t)+H(t) & A^{\rho}(t) X^{\rho}(t)+H^{\rho}(t) \\
A^{\sigma}(t) \tilde{X}^{\sigma}(t)+H^{\sigma}(t) & A(t) \tilde{X}(t)+H(t)
\end{array}\right] } \\
& \times\left[\begin{array}{c}
\alpha \\
1-\alpha
\end{array}\right], \quad t \neq t_{s}, \\
\tilde{\nabla} \dot{X}(t)= & B_{s} \dot{X}(t), \quad t=t_{s}, \quad \dot{X}\left(t_{0}\right)=X_{0} \in \mathbb{Q}^{n \times n},
\end{aligned}\right.
$$

where $A, H: \mathbb{T} \rightarrow \mathbb{Q}^{n \times n}, B_{s} \in \mathbb{Q}^{n \times n}, 0 \leq \alpha \leq 1, t_{s}<t_{s+1}, s \in \mathbb{Z}$ and

$$
\tilde{\nabla} \dot{X}(t)=\left\{\begin{array}{l}
\dot{X}\left(\sigma\left(t^{+}\right)\right)-\dot{X}(t), \quad t=t_{s}, \quad s>0 \\
\dot{X}\left(\rho\left(t^{-}\right)\right)-\dot{X}(t), \quad t=t_{s}, \quad s<0 .
\end{array}\right.
$$

Remark 4.1. For the impulsive term $(2), \tilde{\nabla} \dot{X}(t)=\dot{X}\left(t^{+}\right)-\dot{X}(t)$ for the right dense point $t_{s}(s>0) ; \tilde{\nabla} \dot{X}(t)=\dot{X}(\sigma(t))-\dot{X}(t)$ for the right scattered point $t_{s}(s>0)$. Similarly, $\tilde{\nabla} \dot{X}(t)=\dot{X}\left(t^{-}\right)-\dot{X}(t)$ for the left dense point $t_{s}$ $(s<0) ; \tilde{\nabla} \dot{X}(t)=\dot{X}(\rho(t))-\dot{X}(t)$ for the left scattered point $t_{s}(s<0)$.

Theorem 4.1. For the quaternion impulsive matrix dynamic equation (1), if there exist finite number of points $t_{s}$ in any compact interval $[a, b]_{\mathbb{T}}$ with $a<b$, and the matrices $I+B_{s}$ are nonsingular for all $s \in \mathbb{Z}$. Then a fundamental solution matrix of (1) is given by

$$
\begin{gathered}
\Phi_{A, H}=\left\{\begin{array}{l}
\dot{e}_{A}\left(t, \rho\left(t_{-s}^{-}\right)\right)\left[\prod_{r=-s+1}^{-1}\left(I+B_{r-1}\right) \dot{e}_{A}\left(t_{r-1}, \rho\left(t_{r}^{-}\right)\right)\left(I+B_{-1}\right) \dot{e}_{A}\left(t_{-1}, t_{0}\right) X_{0}\right. \\
+\sum_{v=-1}^{-s+2} \prod_{l=-s+1}^{v-1}\left(I+B_{l-1}\right) \dot{e}_{A}\left(t_{l-1}, \rho\left(t_{l}^{-}\right)\right)\left(I+B_{v-1}\right) \mathbb{E}_{A, H}\left(t_{v-1}, \rho\left(t_{v}^{-}\right)\right) \\
\left.+(I+B-s) \mathbb{E}_{A, H}\left(t_{-s}, \rho\left(t_{-s+1}^{-}\right)\right)\right]+\mathbb{E}_{A, H}\left(t, \rho\left(t_{-s}^{-}\right)\right), \rho\left(t_{-s-1}^{-}\right)<t<\rho\left(t_{-s}^{-}\right),
\end{array}\right. \\
\Phi_{A, H}=\left\{\begin{array}{l}
\prod_{r=-s+1}^{-1}\left(I+B_{r-1}\right) \dot{e}_{A}\left(t_{r-1}, \rho\left(t_{r}^{-}\right)\right)\left(I+B_{-1}\right) \dot{e}_{A}\left(t_{-1}, t_{0}\right) X_{0} \\
+\sum_{v=-1}^{-s+2} v-1 \\
+\left(I+\prod_{-s+1}\right) \mathbb{E}_{A, H}\left(t_{-s}, \rho\left(t_{-s+1}^{-}\right)\right), t=\rho\left(t_{-s}^{-}\right),
\end{array}\right.
\end{gathered}
$$


$\Phi_{A, H}=\left\{\begin{array}{l}\dot{e}_{A}\left(t, t_{0}\right) X_{0}+\mathbb{E}_{A, H}\left(t, t_{0}\right), \quad \rho\left(t_{-1}^{-}\right)<t<\sigma\left(t_{1}^{+}\right), \\ \prod_{r=s-1}^{1}\left(I+B_{r+1}\right) \dot{e}_{A}\left(t_{r+1}, \sigma\left(t_{r}^{+}\right)\right)\left(I+B_{1}\right) \dot{e}_{A}\left(t_{1}, t_{0}\right) X_{0}+\sum_{v=1}^{s-2} \prod_{l=s-1}^{v+1}\left(I+B_{l+1}\right) \\ \times \dot{e}_{A}\left(t_{l+1}, \sigma\left(t_{l}^{+}\right)\right)\left(I+B_{v+1}\right) \mathbb{E}_{A, H}\left(t_{v+1}, \sigma\left(t_{v}^{+}\right)\right)+\left(I+B_{s}\right) \mathbb{E}_{A, H}\left(t_{s}, \sigma\left(t_{s-1}^{+}\right)\right), \\ t=\sigma\left(t_{s}^{+}\right),\end{array}\right.$

and

$$
\Phi_{A, H}=\left\{\begin{array}{l}
\dot{e}_{A}\left(t, \sigma\left(t_{s}^{+}\right)\right)\left[\prod_{r=s-1}^{1}\left(I+B_{r+1}\right) \dot{e}_{A}\left(t_{r+1}, \sigma\left(t_{r}^{+}\right)\right)\left(I+B_{1}\right) \dot{e}_{A}\left(t_{1}, t_{0}\right) X_{0}\right. \\
+\sum_{v=1}^{s-2} \prod_{l=s-1}^{v+1}\left(I+B_{l+1}\right) e_{A}\left(t_{l+1}, \sigma\left(t_{l}^{+}\right)\right)\left(I+B_{v+1}\right) \mathbb{E}_{A, H}\left(t_{v+1}, \sigma\left(t_{v}^{+}\right)\right) \\
\left.+\left(I+B_{s}\right) \mathbb{E}_{A, H}\left(t_{s}, \sigma\left(t_{s-1}^{+}\right)\right)\right]+\mathbb{E}_{A, H}\left(t, \sigma\left(t_{s}^{+}\right)\right), \quad \sigma\left(t_{s}^{+}\right)<t<\sigma\left(t_{s+1}^{+}\right),
\end{array}\right.
$$

where $\mathbb{E}_{A, H}(t, y)=\alpha \int_{y}^{t} e_{A}(t, \sigma(\tau)) H(\tau) \Delta \tau+(1-\alpha) \int_{y}^{t} \hat{e}_{A}(t, \tau) H^{\sigma}(\tau) \Delta \tau, 0 \leq$ $\alpha \leq 1$.

Proof. For $s>0$, by Theorem 3.4, one can obtain

$$
\dot{X}(t)=\left\{\begin{array}{l}
\dot{e}_{A}\left(t, t_{0}\right) X_{0}+\mathbb{E}_{A, H}\left(t, t_{0}\right), \quad t_{0}<t<\sigma\left(t_{1}^{+}\right), \\
\left(I+B_{s}\right) X\left(t_{s}\right), \quad t=\sigma\left(t_{s}^{+}\right), \\
\dot{e}_{A}\left(t, \sigma\left(t_{s}^{+}\right)\right) X\left(\sigma\left(t_{s}^{+}\right)\right)+\mathbb{E}_{A, H}\left(t, \sigma\left(t_{s}^{+}\right)\right), \sigma\left(t_{s}^{+}\right)<t<\sigma\left(t_{s+1}^{+}\right) .
\end{array}\right.
$$

Similarly, for $s<0$, we have

$$
\dot{X}(t)=\left\{\begin{array}{l}
\dot{e}_{A}\left(t, t_{0}\right) X_{0}+\mathbb{E}_{A, H}\left(t, t_{0}\right), \quad \rho\left(t_{-1}^{-}\right)<t<t_{0}, \\
\left(I+B_{s}\right) X\left(t_{s}\right), \quad t=\rho\left(t_{s}^{-}\right), \\
\dot{e}_{A}\left(t, \rho\left(t_{s}^{-}\right)\right) X\left(\rho\left(t_{s}^{-}\right)\right)+\mathbb{E}_{A, H}\left(t, \rho\left(t_{s}^{-}\right)\right), \quad \rho\left(t_{s-1}^{-}\right)<t<\rho\left(t_{s}^{-}\right) .
\end{array}\right.
$$

Hence (3) is a fundamental solution matrix of (1). The proof is completed.

In the following example, we will demonstrate all elements which are required to calculate the fundamental solution matrix of (1) given by (3) on different types of time scales.

Example 4.1. In (3), for $h>0, q>1,0 \leq \alpha \leq 1, t_{s}>t_{s_{1}}$, we have

$$
\dot{e}_{A}\left(t_{s}, t_{s_{1}}\right)=\left\{\begin{array}{l}
I+\sum_{n=1}^{\infty} \int_{t_{s_{1}}}^{t} A\left(t_{n}\right) \int_{t_{s_{1}}}^{t_{n}} A\left(t_{n-1}\right) \ldots \int_{t_{s_{1}}}^{t_{2}} A\left(t_{1}\right) \mathrm{d} t_{1} \ldots \mathrm{d} t_{n-1} \mathrm{~d} t_{n}, \mathbb{T}=\mathbb{R}, \\
\alpha\left(I+\frac{t_{s}-t_{s_{1}}}{\sum_{n=1}^{h}} h^{n} \prod_{\tilde{n}=1}^{n-1} A\left(t_{s}-\tilde{n} h\right) \frac{t_{s}-t_{s_{1}}}{h} \sum_{n=0}{ }_{n=1} A\left(t_{s_{1}}+n h\right)\right)+(1-\alpha)(I \\
\left.+\sum_{n=1}^{\infty} \int_{t_{s_{1}}}^{t_{s}} A^{\sigma}\left(t_{n}\right) \ldots \int_{t_{s_{1}}}^{\sigma\left(t_{2}\right)} A^{\sigma}\left(t_{1}\right) \Delta t_{1} \ldots \Delta t_{n}\right), \quad \mathbb{T}=h \mathbb{Z},
\end{array}\right.
$$


$\dot{e}_{A}\left(t_{s}, t_{s_{1}}\right)=\left\{\begin{array}{l}\alpha\left(I+\sum_{n=1}^{\frac{\ln t_{s}-\ln t_{s_{1}}}{\ln q}} \frac{(q-1)^{n} t_{s}^{n-1}}{q^{\frac{n(n-1)}{2}}} \prod_{\tilde{n}=1}^{n-1} A\left(t_{s} q^{-\tilde{n}}\right)\right. \\ +(1-\alpha)\left(I+\sum_{n=1}^{\infty} \int_{t_{s_{1}}}^{t_{s}} A^{\sigma}\left(t_{n}\right) \ldots \int_{t_{s_{1}}}^{\sigma\left(t_{2}\right)} A^{\sigma}\left(t_{1}\right) \Delta t_{1} \ldots \Delta t_{n}\right), \quad \mathbb{T}=\overline{q^{\mathbb{Z}} t_{s}-\ln t_{s_{1}}}-(n-2) \\ \int_{t_{s_{1}}}^{t_{s}}\left(I+\sum_{n=1}^{\infty} \int_{\tau}^{t} A\left(t_{n}\right) \ldots \int_{\tau}^{t_{2}} A\left(t_{1}\right) \mathrm{d} t_{1} \ldots \mathrm{d} t_{n}\right) H(\tau) \mathrm{d} \tau, \mathbb{T}=\mathbb{R},\end{array}\right.$

and

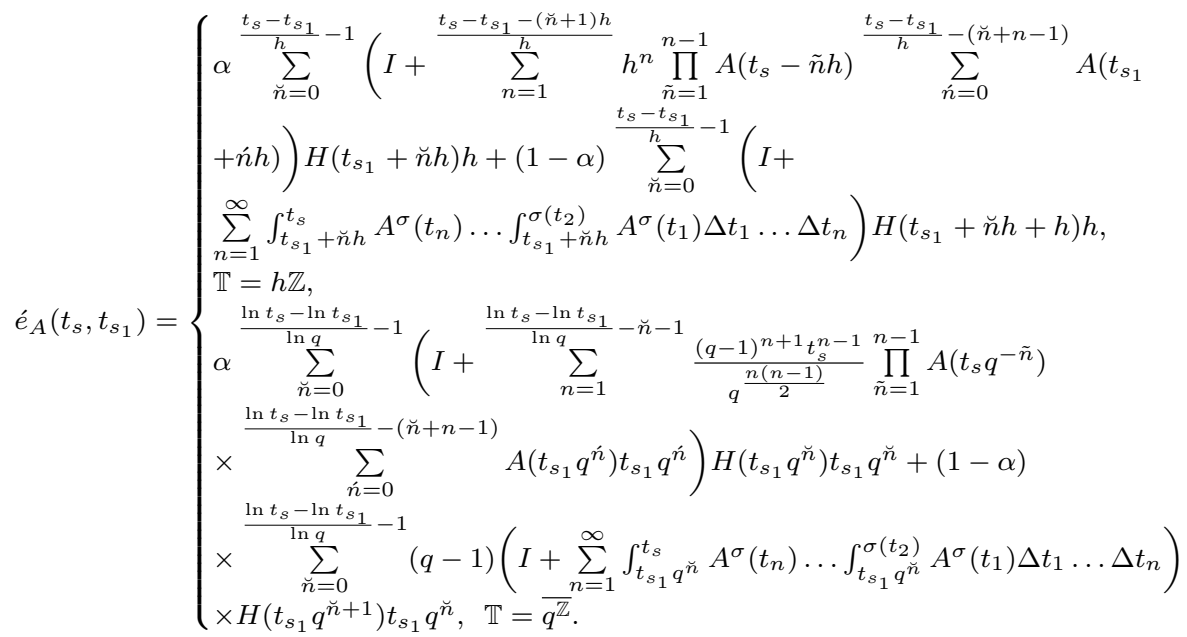

For $t_{s}<t_{s_{1}}$, we have

$$
\begin{aligned}
& \dot{e}_{A}\left(t_{s}, t_{s_{1}}\right)=\left\{\begin{array}{l}
I+\sum_{n=1}^{\infty} \int_{t_{s_{1}}}^{t} A\left(t_{n}\right) \int_{t_{s_{1}}}^{t_{n}} A\left(t_{n-1}\right) \ldots \int_{t_{s_{1}}}^{t_{2}} A\left(t_{1}\right) \mathrm{d} t_{1} \ldots \mathrm{d} t_{n-1} \mathrm{~d} t_{n}, \mathbb{T}=\mathbb{R}, \\
\alpha\left(I+\sum_{n=1}^{\infty} \int_{t_{s_{1}}}^{t_{s}} A\left(t_{n}\right) \int_{t_{s_{1}}}^{t_{n}} A\left(t_{n-1}\right) \ldots \int_{t_{s_{1}}}^{t_{2}} A\left(t_{1}\right) \Delta t_{1} \ldots \Delta t_{n-1} \Delta t_{n}\right) \\
+(1-\alpha)\left(I+\sum_{n=1}^{\mid \frac{t_{s}-t_{s_{1}} \mid}{h}}(-h)^{n} \prod_{\tilde{n}=1}^{n-1} A\left(t_{s}+\tilde{n} h\right) \sum_{n=0}^{\left|\frac{t_{s}-t_{s_{1}}}{h}\right|-(n-2)} A\left(t_{s_{1}}-\tilde{n} h\right)\right), \\
\mathbb{T}=h \mathbb{Z},
\end{array}\right. \\
& \dot{e}_{A}\left(t_{s}, t_{s_{1}}\right)=\left\{\begin{array}{l}
\alpha\left(I+\sum_{n=1}^{\infty} \int_{t_{s_{1}}}^{t_{s}} A\left(t_{n}\right) \int_{t_{s_{1}}}^{t_{n}} A\left(t_{n-1}\right) \ldots \int_{t_{s_{1}}}^{t_{2}} A\left(t_{1}\right) \Delta t_{1} \ldots \Delta t_{n-1} \Delta t_{n}\right) \\
+(1-\alpha)\left(I+\sum_{n=1}^{\left|\frac{\ln t_{s}-\ln t_{s_{1}}}{\ln q}\right|}(1-q)^{n} t_{s}^{n-1} q^{\frac{(n-2)(n-1)}{2}} \prod_{\tilde{n}=1}^{n-1} A\left(t_{s} q^{\tilde{n}}\right)\right. \\
\left.\times \frac{\left|\frac{\ln t_{s}-\ln t_{s_{1}}}{\ln q}\right|-(n-2)}{\sum_{n=0}} A\left(t_{s_{1}} q^{-\dot{n}}\right) t_{s_{1}} q^{-\dot{n}-1}\right), \quad \mathbb{T}=\overline{q^{\mathbb{Z}}}
\end{array}\right.
\end{aligned}
$$




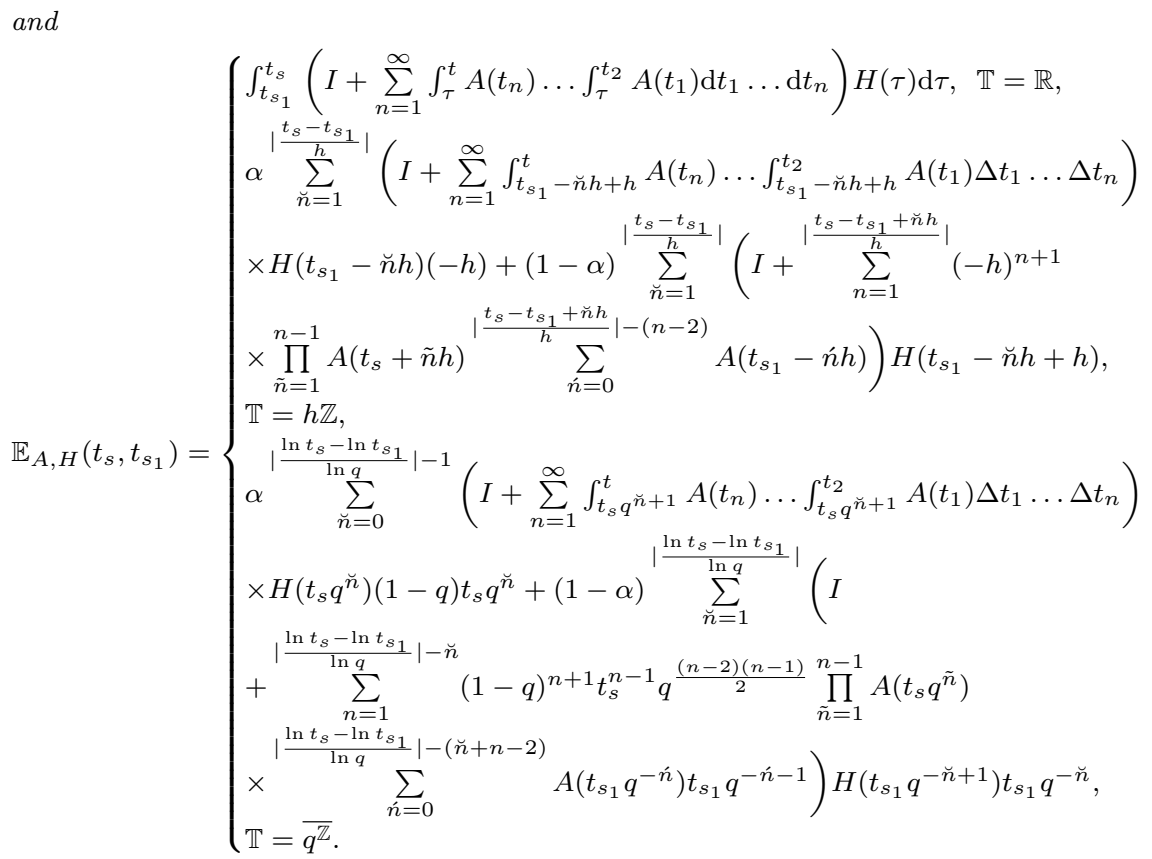

Example 4.2. In (3), let $\mathbb{T}=\mathbb{N}, t_{s}=3 m-1, m \in \mathbb{N}^{+}$,

$A(t)=H(t-2)=\left[\begin{array}{cc}\frac{1}{4^{t}}+i \frac{(-1)^{t}}{4^{t}}+j \frac{\sin \left(t \pi+\frac{\pi}{2}\right)}{4^{t}} & \frac{1}{4^{t}}+i \frac{(-1)^{t}}{4^{t}}+j \frac{\sin \left(t \pi+\frac{\pi}{2}\right)}{4^{t}} \\ 0 & \frac{1}{4^{t}}+i \frac{\cos (t \pi+\pi)}{4^{t}}+j \frac{\tan \frac{(-1)^{t+1}}{4^{t}} \pi}{4^{t}}\end{array}\right]$.

Then

$$
\begin{aligned}
e_{A}(3 m-1,3 m-3)= & I+A(3 m-3)+A(3 m-2) \\
& +A(3 m-2) A(3 m-3)=L_{1}, \\
\hat{e}_{A}(3 m-1,3 m-3)= & I+\sum_{n=1}^{\infty} \sum_{\grave{n}=0}^{n} A^{\grave{n}}(3 m-1) A^{n-\grave{n}}(3 m-2)=L_{2}, \\
L_{3}= & \int_{3 m-3}^{3 m-1} e_{A}(3 m-1, \sigma(\tau)) H(\tau) \Delta \tau \\
= & H(3 m-3)+A(3 m-2) H(3 m-3)+H(3 m-2), \\
L_{4}= & \int_{3 m-3}^{3 m-1} \hat{e}_{A}(3 m-1, \tau) H^{\sigma}(\tau) \Delta \tau \\
= & \left(I+\sum_{n=1}^{\infty} A^{n}(3 m-1)\right) H(3 m-1)
\end{aligned}
$$




$$
\begin{aligned}
& +\left(I+\sum_{n=1}^{\infty} \sum_{\grave{n}=0}^{n} A^{\grave{n}}(3 m-1) A^{n-\grave{n}}(3 m-2)\right) \\
& \times H(3 m-2),
\end{aligned}
$$

where

$$
\begin{aligned}
& L_{1}= \\
& {\left[\begin{array}{cc}
1+\frac{3}{4^{6 m-5}}+\frac{5-3(-1)^{3 m-2}(i+j)}{4^{3 m-2}} & \frac{6}{4^{6 m-5}}+\frac{5-3(-1)^{3 m-2}(i+j)}{4^{3 m-2}} \\
0 & 1+\frac{3}{4^{6 m-5}}+\frac{5+3(-1)^{3 m-2}(i+j)}{4^{3 m-2}}
\end{array}\right],} \\
& L_{2} \\
& \sum_{n=1}^{\infty} \sum_{\grave{n}=0}^{n} \frac{1}{4^{3 m n-2 n+\grave{n}}}\left[\begin{array}{cc}
\left(1+(i+j)(-1)^{3 m-1}\right)^{\grave{n}} & \sum_{l=0}^{\grave{n}} 3^{l}\left(1+(i+j)(-1)^{3 m-1}\right)^{\grave{n}-l} \\
0 & \left(1-(i+j)(-1)^{3 m-1}\right)^{\grave{n}}
\end{array}\right] \\
& \times\left[\begin{array}{cc}
\left(1-(i+j)(-1)^{3 m-1}\right)^{n-\grave{n}} & \sum_{l=0}^{n-\grave{n}} 3^{l}\left(1-(i+j)(-1)^{3 m-1}\right)^{n-\grave{n}-l} \\
0 & \left(1+(i+j)(-1)^{3 m-1}\right)^{n-\grave{n}}
\end{array}\right]+I, \\
& L_{3}= \\
& {\left[\begin{array}{cc}
1+\frac{3}{4^{6 m-3}}+\frac{5-3(-1)^{3 m}(i+j)}{4^{3 m}} & \frac{6}{4^{6 m-3}}+\frac{5-3(-1)^{3 m}(i+j)}{4^{3 m}} \\
0 & 1+\frac{3}{4^{6 m-3}}+\frac{5+3(-1)^{3 m}(i+j)}{4^{3 m}}
\end{array}\right],} \\
& L_{4}= \\
& \sum_{n=1}^{\infty} \frac{1}{4^{3 m(n+1)-n+1}}\left[\begin{array}{cc}
\left(1+(i+j)(-1)^{3 m-1}\right)^{n+1} & \sum_{l=0}^{n+1} 3^{l}\left(1+(i+j)(-1)^{3 m-1}\right)^{n+1-l} \\
0 & \left(1-(i+j)(-1)^{3 m-1}\right)^{n+1}
\end{array}\right] \\
& \sum_{n=1}^{\infty} \sum_{\grave{n}=0}^{n} \frac{1}{4^{3 m(n+1)-2 n+\grave{n}}}\left[\begin{array}{cc}
\left(1+(i+j)(-1)^{3 m-1}\right)^{\grave{n}} & \sum_{l=0}^{\grave{n}} 3^{l}\left(1+(i+j)(-1)^{3 m-1}\right)^{\grave{n}-l} \\
0 & \left(1-(i+j)(-1)^{3 m-1}\right)^{\grave{n}}
\end{array}\right] \\
& \times\left[\begin{array}{cc}
\left(1-(i+j)(-1)^{3 m-1}\right)^{n-\grave{n}+1} & \sum_{l=0}^{n-\grave{n}+1} 3^{l}\left(1-(i+j)(-1)^{3 m-1}\right)^{n-\grave{n}-l+1} \\
0 & \left(1+(i+j)(-1)^{3 m-1}\right)^{n-\grave{n}+1}
\end{array}\right] \\
& +\left[\begin{array}{cc}
\frac{5-3(-1)^{3 m+1}(i+j)}{4^{3 m+1}} & \frac{5-3(-1)^{3 m+1}(i+j)}{4^{3 m+1}} \\
0 & \frac{5+3(-1)^{3 m+1}(i+j)}{4^{3 m+1}}
\end{array}\right] \text {. }
\end{aligned}
$$

By Theorem 4.1, the following theorem is immediate.

Theorem 4.2. The cauchy matrix of (1) is given by:

$$
W(t, y)=\left\{\begin{array}{l}
\dot{e}_{A}(t, y)+\mathbb{E}_{A, H}(t, y), t_{s-1}<t, y<t_{s}, t_{-s-1}<t, y<t_{-s}, \\
\dot{e}_{A}\left(t, \sigma\left(t_{s}^{+}\right)\right)\left(I+B_{s}\right)\left[e_{A}\left(t_{s}, y\right)+\mathbb{E}_{A, H}\left(t_{s}, y\right)\right]+\mathbb{E}_{A, H}\left(t, \sigma\left(t_{s}^{+}\right)\right), \\
t_{s-1}<y \leq t_{s}<t \leq t_{s+1}, \\
\dot{e}_{A}\left(t, t_{s}\right)\left(I+B_{s}\right)^{-1}\left[\dot{e}_{A}\left(\sigma\left(t_{s}^{+}\right), y\right)+\mathbb{E}_{A, H}\left(\sigma\left(t_{s}^{+}\right), y\right)\right]+\mathbb{E}_{A, H}\left(t, t_{s}\right), \\
t_{s-1}<t \leq t_{s}<y \leq t_{s+1},
\end{array}\right.
$$




$$
\begin{aligned}
& W(t, y)=\left\{\begin{array}{l}
\dot{e}_{A}\left(t, \rho\left(t_{-s}^{-}\right)\right)\left(I+B_{-s}\right)\left[\dot{e}_{A}\left(t_{-s}, y\right)+\mathbb{E}_{A, H}\left(t_{-s}, y\right)\right]+\mathbb{E}_{A, H}\left(t, \rho\left(t_{-s}^{-}\right)\right), \\
t_{-s-1}<t \leq t_{-s}<y \leq t_{-s+1}, \\
e_{A}\left(t, t_{-s}\right)\left(I+B_{-s}\right)-1\left[\dot{e}_{A}\left(\rho\left(t_{-s}^{-}\right), y\right)+\mathbb{E}_{A, H}\left(\rho\left(t_{-s}^{-}\right), y\right)\right]+\mathbb{E}_{A, H}\left(t, t_{-s}\right), \\
t_{-s-1}<y \leq t_{-s}<t \leq t_{-s+1},
\end{array}\right.
\end{aligned}
$$

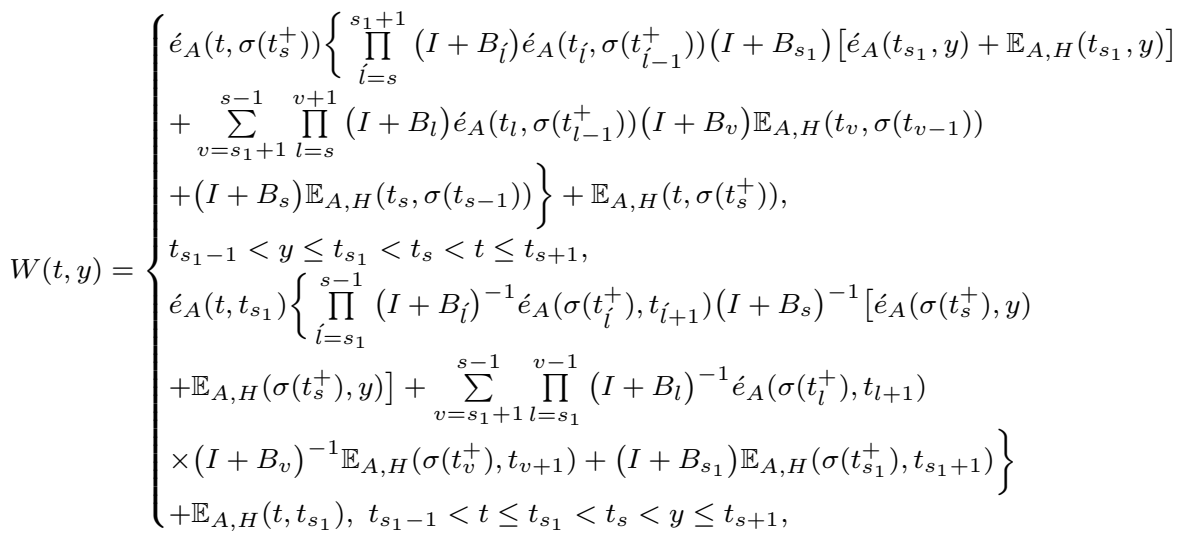

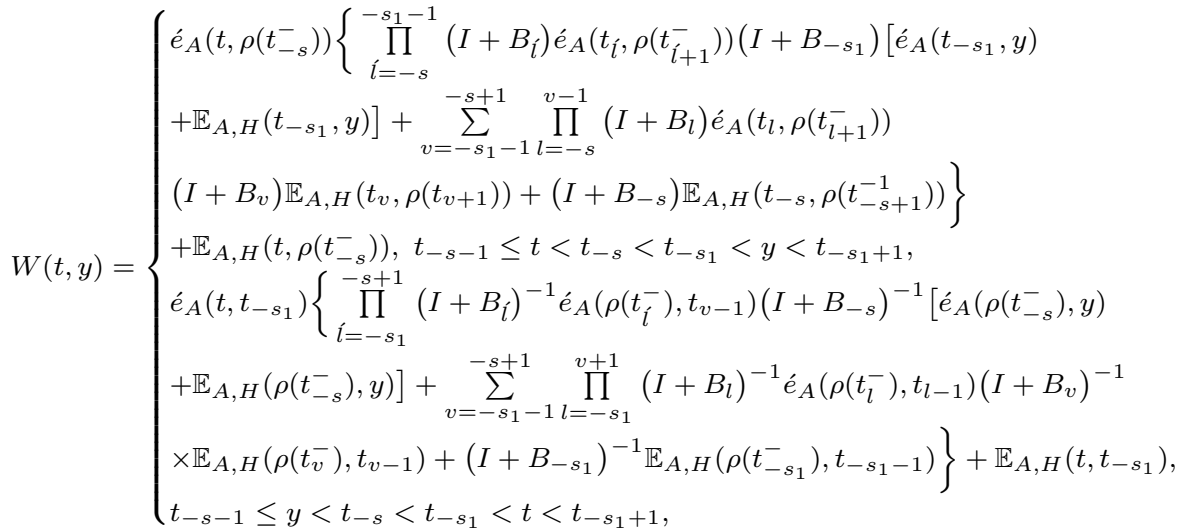

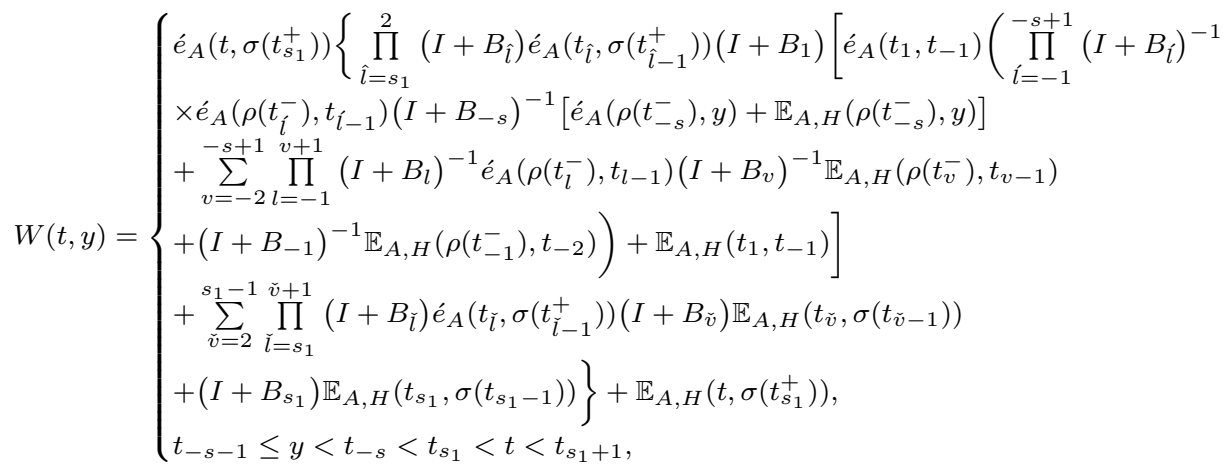


and

$$
W(t, y)=\left\{\begin{array}{l}
e_{A}\left(t, \rho\left(t_{-s}^{-}\right)\right)\left\{\prod _ { \hat { l } = - s } ^ { - 2 } ( I + B _ { \hat { l } } ) \dot { e } _ { A } ( t _ { \hat { l } } , \rho ( t _ { \hat { l } + 1 } ^ { - } ) ) ( I + B _ { - 1 } ) \left[e _ { A } ( t _ { - 1 } , t _ { 1 } ) \left(\prod_{i=1}^{s_{1}-1}(I\right.\right.\right. \\
\left.+B_{i}\right)^{-1} e_{A}\left(\sigma\left(t_{i}^{+}\right), t_{i+1}\right)\left(I+B_{s_{1}}\right)^{-1}\left[e_{A}\left(\sigma\left(t_{s_{1}}^{+}\right), y\right)+\mathbb{E}_{A, H}\left(\sigma\left(t_{s_{1}}^{+}\right), y\right)\right] \\
+\sum_{v=2}^{s_{1}-1} \prod_{l=1}^{-1}\left(I+B_{l}\right)^{-1} \dot{e}_{A}\left(\sigma\left(t_{l}^{+}\right), t_{l+1}\right)\left(I+B_{v}\right)^{-1} \mathbb{E}_{A, H}\left(\sigma\left(t_{v}^{+}\right), t_{v+1}\right) \\
\left.\left.+\left(I+B_{1}\right) \mathbb{E}_{A, H}\left(\sigma\left(t_{1}^{+}\right), t_{2}\right)\right)+\mathbb{E}_{A, H}\left(t_{-1}, t_{1}\right)\right] \\
+\sum_{\tilde{v}=-2}^{-s+1} \prod_{\tilde{l}=-s}^{\tilde{v}-1}\left(I+B_{\tilde{l}}\right) \dot{e}_{A}\left(t_{\tilde{l}}, \rho\left(t_{\tilde{l}+1}^{-}\right)\right)\left(I+B_{\tilde{v}}\right) \mathbb{E}_{A, H}\left(t_{\tilde{v}}, \rho\left(t_{\tilde{v}+1}\right)\right)+\left(I+B_{-s}\right) \\
\left.\times \mathbb{E}_{A, H}\left(t_{-s}, \rho\left(t_{-s+1}^{-1}\right)\right)\right\}+\mathbb{E}_{A, H}\left(t, \rho\left(t_{-s}^{-}\right)\right), t_{-s-1} \leq t<t_{-s}<t_{s_{1}}<y<t_{s_{1}+1},
\end{array}\right.
$$

where $\mathbb{E}_{A, H}(t, y)=\alpha \int_{y}^{t} e_{A}(t, \sigma(\tau)) H(\tau) \Delta \tau+(1-\alpha) \int_{y}^{t} \hat{e}_{A}(t, \tau) H^{\sigma}(\tau) \Delta \tau, 0 \leq$ $\alpha \leq 1, s \in \mathbb{N}^{+}$.

\section{References}

[1] S. Adler, Quaternionic Quantum Mechanics and Quantum Fields. Oxford University Press, New York, 1994.

[2] R.P. Agarwal, C. Wang, D. O'Regan, Recent development of time scales and related topics on dynamic equations. Mem. Differential Equations Math. Phys. 67 (2016) 131-135.

[3] F.M. Atici, G.Sh. Guseinov, On Green's functions and positive solutions for boundary value problems on time scales, J. Comput. Appl. Math. 18, (2002) 75-99.

[4] M. Bohner, A. Peterson, Dynamic Equations on Time Scales, An Introduction with Applications. Birkhauser, Boston, 2001.

[5] Z. Cai, K.I. Kou, Laplace transform: a new approach in solving linear quaternion differential equations, Math. Meth. Appl. Sci. 41, (2018) 40334048 .

[6] D. Cheng, K.I. Kou, Y.H. Xia, A unified analysis of linear quaternion dynamic equations on time scales, J. Appl. Anal. Comput. 8, (2018) 172201.

[7] D.J. Gibbon, D.D. Holm, R.M. Kerr, I. Roulstone, Quaternions and particle dynamics in the Euler fluid equations, Nonlinearity, 19, (2006) 19691983. 
[8] W.R. Hamilton, Elements of Quaternions. London, UK: Longmans, Green, \& Co, 1866.

[9] S. Hilger, Ein Maßkettenkalkül mit Anwendung auf Zentrumsmannigfaltigkeiten, Ph.D. Thesis, Universität Würzburg, 1988.

[10] K.I. Kou, Y.H. Xia, Linear quaternion differential equations: basic theory and fundamental results, Stud. Appl. Math. 141, (2018) 3-45.

[11] V. Lakshmikantham, D.D. Bainov, P.S. Simeonov, Theory of Impulsive Differential Equations, World Scientific, Singapore, 1989.

[12] Z. Li, C. Wang, R.P. Agarwal, The non-eigenvalue form of Liouville's formula and $\alpha$-matrix exponential solutions for combined matrix dynamic equations on time scales, Mathematics, 7(10), 962; https://doi.org/10.3390/math7100962 (2019).

[13] Z. Li, C. Wang, R.P. Agarwal, D. O'Regan, Commutativity of quaternionmatrix-valued functions and quaternion matrix dynamic equations on time scales, Stud. Appl. Math., 146, (2021) 139-210.

[14] Z. Li, C. Wang, R.P. Agarwal, R. Sakthivel, Hyers-Ulam-Rassias stability of quaternion multidimensional fuzzy nonlinear difference equations with impulses, Iranian J. Fuzzy Syst., 10.22111/IJFS.2021.5950 (2021).

[15] Z. Li, C. Wang, Cauchy matrix and Liouville formula of quaternion impulsive dynamic equations on time scales, Open Math., 18, (2020) 353-377.

[16] G. Qin, C. Wang, Lebesgue-Stieltjes combined $\diamond_{\alpha}$-measure and integral on time scales, Rev. R. Acad. Cienc. Exactas Fís. Nat. Ser. A Mat. RACSAM. https://doi.org/10.1007/s13398-021-01000-y, 115:50 (2021).

[17] J.W. Rogers, Q. Sheng, Notes on the diamond- $\alpha$ dynamic derivative on time scales, J. Math. Anal. Appl. 326, (2007) 228-241.

[18] Q. Sheng, M. Fadag, J. Henderson, J.M. Davis, An exploration of combined dynamic derivatives on time scales and their applications, Nonlinear Anal.: RWA. 7, (2006) 395-413.

[19] C. Wang, R.P. Agarwal, Almost periodic dynamics for impulsive delay neural networks of a general type on almost periodic time scales, Commun. Nonlinear Sci. Numer. Simulat. 36, (2016) 238-251.

[20] C. Wang, R.P. Agarwal, D. O'Regan, R. Sakthivel, A computation method of Hausdorff distance for translation time scales, 99, (2020) 12181247 . 
[21] C. Wang, R.P. Agarwal, Relatively dense sets, corrected uniformly almost periodic functions on time scales, and generalizations, Adv. Differ. Equa. 2015:312, (2015) 1-9.

[22] C. Wang, R.P. Agarwal, D. O’Regan, Periodicity, almost periodicity for time scales and related functions. Nonauton. Dyn. Syst. 3, (2016) 24-41.

[23] C. Wang, R.P. Agarwal, D. O'Regan, $\delta$-almost periodic functions and applications to dynamic equations, Mathematics 7, 525, doi.org/10.3390/math7060525 (2019).

[24] C. Wang, R. Sakthivel, G.M. N'Guérékata, S-almost automorphic solutions for impulsive evolution equations on time scales in shift operators, Mathematics, 8(6), 1028; https://doi.org/10.3390/math8061028 (2020).

[25] C. Wang, R.P. Agarwal and D. O'Regan, Calculus of fuzzy vectorvalued functions and almost periodic fuzzy vector-valued functions on time scales, Fuzzy. Sets and Syst., 375 (2019) 1-52.

[26] C. Wang, Almost periodic solutions of impulsive BAM neural networks with variable delays on time scales. Commun. Nonlinear Sci. Numer. Simul. 19, (2014) 2828-2842.

[27] C. Wang, R.P. Agarwal, A Further study of almost periodic time scales with some notes and applications Abstr. Appl. Anal., Article ID 267384, (2014) 1-12.

[28] C. Wang, R.P. Agarwal, Weighted piecewise pseudo almost automorphic functions with applications to abstract impulsive $\nabla$-dynamic equations on time scales, Adv. Differ. Equa. 2014:153, (2014) 1-29.

[29] C. Wang, R.P. Agarwal, D. O'Regan, П-semigroup for invariant under translations time scales and abstract weighted pseudo almost periodic functions with applications. Dyn. Syst. Appl. 25, (2016) 1-28.

[30] C. Wang, R.P. Agarwal, A classification of time scales and analysis of the general delays on time scales with applications. Math. Meth. Appl. Sci. 39, (2016) 1568-1590.

[31] C. Wang, R.P. Agarwal, Almost automorphic functions on semigroups induced by complete-closed time scales and application to dynamic equations, Discret. Continu. Dynam. Syst. B. 25, (2020) 781-798.

[32] C. Wang, R.P. Agarwal, D. O'Regan, R. Sakthivel, Local pseudo almost automorphic functions with applications to semilinear dynamic 
equations on changing-periodic time scales, Bound Value Probl. 133, doi:10.1186/s13661-019-1247-4 (2019).

[33] C. Wang, R.P. Agarwal, D. O'Regan, Local-periodic solutions for functional dynamic equations with infinite delay on changing-periodic time scales, Math. Slovaca. 68, (2018) 1397-1420.

[34] C. Wang, R.P. Agarwal, Changing-periodic time scales and decomposition theorems of time scales with applications to functions with local almost periodicity and automorphy, Adv. Differ. Equa. 2015:296 (2015) 1-21.

[35] C. Wang, R.P. Agarwal, D. O'Regan, A matched space for time scales and applications to the study on functions, Adv. Differ. Equa. 2017:305, (2017) 1-28.

[36] C. Wang, R.P. Agarwal, D. O'Regan, $n_{0}$-order $\Delta$-almost periodic functions and dynamic equations, Applic. Anal. 97, (2018) 2626-2654.

[37] C. Wang, R.P. Agarwal, D. O'Regan, Weighted pseudo $\delta$-almost automorphic functions and abstract dynamic equations, Georgian Math. J. doi: https://doi.org/10.1515/gmj-2019- 2066 (2019) (In press).

[38] C. Wang, R.P. Agarwal, R. Sakthivel, Almost periodic oscillations for delay impulsive stochastic Nicholson's blowflies timescale model, Comput. Appl. Math. 37, (2018) 3005-3026.

[39] C. Wang, R.P. Agarwal, Almost periodic solution for a new type of neutral impulsive stochastic Lasota-Wazewska timescale model, Appl. Math. Lett. 70, (2017) 58-65.

[40] C. Wang, R. Sakthivel, Double almost periodicity for high-order Hopfield neural networks with slight vibration in time variables, Neurocomputing, $282,(2018) 1-15$.

[41] C. Wang, R.P. Agarwal, D. O'Regan, R. Sakthivel, Theory of Translation Closedness for Time Scales, Developments in Mathematics, Vol. 62, Springer, Switzerland, 2020.

[42] C. Wang, G. Qin, R.P. Agarwal, D. O’Regan, $\nabla_{\alpha}$-Measurability and combined measure theory on time scales, Applic. Anal., https://doi.org/10.1080/00036811.2020.1820997, 2020 (In press).

[43] C. Wang, R.P. Agarwal, D. O'Regan, Matrix measure on time scales and almost periodic analysis of the impulsive Lasota-Wazewska model with patch structure and forced perturbations, Math. Meth. Appl. Sci. 39, (2016) 5651-5669. 
[44] C. Wang, Piecewise pseudo almost periodic solution for impulsive nonautonomous highorder Hopfield neural networks with variable delays, Neurocomputing, 171, (2016) 1291-1301.

[45] C. Wang, R.P. Agarwal, Uniformly rd-piecewise almost periodic functions with applications to the analysis of impulsive $\Delta$-dynamic system on time scales, Appl. Math. Comput. 259, (2015) 271-292.

[46] C. Wang, R.P. Agarwal, D. O'Regan, Weighted piecewise pseudo doublealmost periodic solution for impulsive evolution equations. J. Nonlinear Sci. Appl. 10, (2017) 3863-3886.

[47] C. Wang, R.P. Agarwal, D. O'Regan, Compactness criteria and new impulsive functional dynamic equations on time scales, Adv. Differ. Equa. 2016:197, (2016) 1-41.

[48] C. Wang, R.P. Agarwal, Exponential dichotomies of impulsive dynamic systems with applications on time scales, Math. Meth. Appl. Sci. 38, (2015) 3879-3900.

[49] C. Wang, R.P. Agarwal, D. O'Regan, R. Sakthivel, Discontinuous generalized double-almost- periodic functions on almost-complete-closed time scales, Bound Value Probl. 165, doi.org/10.1186/s13661-019-1283-0 (2019).

[50] C. Wang, Z. Li, R. P. Agarwal, D. O'Regan, Coupled-jumping timescale theory and applications to time-hybrid dynamic equations, convolution and Laplace transforms. Dynam. Syst. Appl., 30 (2021) 461-508.

[51] P. Wilczynski, Quaternionic-valued ordinary differential equations. The Riccati equation, J. Differ. Equ. 247, (2009) 2163-2187.

[52] F. Zhang, Quaternions and matrices of quaternions, Linear Algebra and its Applications, 251, (1997) 21-57.

[53] J. Zhu, J. Sun, Existence and uniqueness results for quaternion-valued nonlinear impulsive differential systems, J. Syst. Sci. Compl. 31, (2018) 596-607.

[54] J. Zhu, J. Sun, Global exponential stability of Clifford-valued recurrent neural networks, Neurocomputing, 173, (2016) 685-689. 
Chao Wang,

Department of Mathematics,

Yunnan University,

Kunming, Yunnan 650091, China.

Email: chaowang@ynu.edu.cn

Zhien Li,

Department of Mathematics,

Yunnan University,

Kunming, Yunnan 650091, China.

Email: lizhienyunnan@163.com

Ravi P. Agarwal,

Department of Mathematics,

Texas A\&M University-Kingsville,

TX 78363-8202, Kingsville, TX, USA.

Email: ravi.agarwal@tamuk.edu 